\title{
Poil de Carotte et ses traductions italiennes au féminin : l'évolution d'un classique littéraire
}

\section{Chiara Elefante}

\section{(2) OpenEdition Journals}

Édition électronique

URL : https://journals.openedition.org/dhfles/3329

DOI : $10.4000 /$ dhfles.3329

ISSN : 2221-4038

Éditeur

Société Internationale pour l'Histoire du Français Langue Étrangère ou Seconde

Édition imprimée

Date de publication : 1 juin 2012

ISSN : 0992-7654

Référence électronique

Chiara Elefante, «Poil de Carotte et ses traductions italiennes au féminin : l'évolution d'un classique littéraire », Documents pour I'histoire du français langue étrangère ou seconde [En ligne], 47-48 | 2012, mis en ligne le 01 janvier 2015, consulté le 26 mars 2023. URL : http://journals.openedition.org/dhfles/ 3329 ; DOI : https://doi.org/10.4000/dhfles.3329

Ce document a été généré automatiquement le 26 mars 2023

Tous droits réservés 


\title{
Poil de Carotte et ses traductions italiennes au féminin : l'évolution d'un classique littéraire
}

\author{
Chiara Elefante
}

\section{La littérature de jeunesse en tant que lieu d'accès des femmes à l'écriture et à la traduction}

1 En analysant la littérature de jeunesse ${ }^{1}$ suivant un parcours diachronique, en France comme en Italie, on s'aperçoit d'emblée qu'il s'agit d'un univers de la création artistique qui n'a été reconnu et apprécié à sa juste valeur que tout récemment. Si les différentes approches disciplinaires se sont occupées de ces typologies textuelles en partant de plusieurs points de vue, de celui pédagogique jusqu'à celui éditorial, en passant par la perspective plus strictement littéraire, un aspect semble pourtant faire l'unanimité, à savoir le fait que " la littérature de jeunesse fut historiquement un lieu d'accès des femmes à l'écriture et elle est un des volets de l'histoire de l'écriture féminine » (Nières-Chevrel 2009: 80). Nières-Chevrel remarque également qu'il s'agit encore aujourd'hui d'une branche de la littérature où l'écriture féminine se singularise et mérite une attention particulière. Il suffit de penser aux études que les gender studies ont consacrées, depuis quelque temps déjà, non seulement à certains textes de la littérature de jeunesse en dénonçant leur nature misogyne, mais également aux textes les plus récents en mettant en valeur leurs nouvelles représentations du féminin, pour pouvoir en déduire qu'au sein de la production de l'«extrême contemporain" l'écriture féminine a acquis un poids important et suscité un intérêt considérable. Nous pouvons étendre ces considérations au domaine de cette forme singulière de l'écriture qu'est la traduction : de ce point de vue, au cours de l'histoire, on a accepté que «les femmes s'adonnent à la traduction, car ce sont les idées d'un autre, le plus souvent celles d'un homme, qu'elles réexpriment et non leurs propres idées » (Delisle $2002:$ 7). Si dans l'histoire passée de la traduction de la littérature de jeunesse on ne saurait sans 
doute parler d'une présence majoritaire de traductrices, il n'en demeure pas moins qu'en Europe les cas de femmes ayant débuté précisément par cette forme de traduction pour se consacrer ensuite à l'écriture sont assez nombreux. Cette tendance se perpétue encore aujourd'hui, tout au moins dans les panoramas français et italien : pour ne citer que quelques noms, il suffit de penser, en France, à Francine Bouchet, qui traduit l'écrivaine italienne Giusi Quarenghi, et qui publie en même temps des textes personnels destinés aux enfants. Quant au domaine éditorial italien, nous pouvons constater qu'Anna Sarfatti et Beatrice Masini, deux écrivaines parmi les plus reconnues dans le domaine de l'écriture pour les jeunes, sont également deux grandes traductrices de l'anglais, la première du Docteur Seuss, la deuxième de la saga de Harry Potter.

Si l'on passe ensuite, et ce sera en fait le sujet de cette étude, au monde des classiques, la question se fait plus complexe : s'agissant de textes qui, du point de vue éditorial, peuvent éventuellement s'adresser à un public de jeunes comme à un public d'adultes, le choix du traducteur ou de la traductrice suit d'autres voies, qui relèvent aussi, parfois, de logiques strictement éditoriales : sans vouloir trop généraliser, rappelons néanmoins que l'une des traductions les plus récentes et les plus connues de La guerre des boutons est due à une femme, Angela Nanetti (Pergaud 2003) et celle de Sans famille est l'œuvre de Rossana Guarnieri (Malot 2001), qui juxtaposent encore une fois, toutes les deux, l'expérience de la traduction à celle de l'écriture.

3 La présence grandissante de traductrices dans ce domaine éditorial peut être interprétée de plusieurs manières : on peut tout d'abord évoquer la solidarité manifeste entre les traductrices et les femmes écrivaines, mais on peut également envisager une sorte de contiguïté entre les femmes qui traduisent et les enfants auxquels elles s'adressent, qui ont fait pendant longtemps partie de ce que Susanne de Lotbinière définissait « le groupe muetté »:

Loin d'être neutre, l'acte de traduire constitue une prise de parole pleine de conséquences. En plus d'être une voie de passage d'une langue à une autre, la traduction est aussi un lieu de pouvoir. Pour les traductrices [...] elle représente un espace à investir, un pouvoir à exercer. [...] Font partie du groupe muetté : les enfants, les minorités visibles et invisibles, bref tout groupe ou individu dont la parole est reléguée au sous-sol muet de l'ordre social. (Lotbinière 1991 : 12-14)

On peut encore reconnaître, parmi les raisons qui sollicitent aujourd'hui les principaux éditeurs de jeunesse à miser sur la traduction féminine, la capacité des femmes à vivre à l'intersection entre l'univers adulte auquel elles appartiennent et l'univers enfantin ou juvénile auquel elles s'adressent. En ce sens les traductrices de littérature de jeunesse pourraient être rapprochées des traductrices de textes émanant de minorités, dont le point de départ en traduction est justement l'abolition de la frontière entre un soi-disant centre et une prétendue marginalité :

La traductrice de textes [...] émanant de minorités doit vivre à cheval entre deux mondes, [...] elle a besoin d'élaborer une notion de frontière qui soit perméable, changeante. Il lui faut jouer et négocier avec de multiples instances. Pour elle il s'agit, après tout, de ne pas méconnaître ni l'altérité collective ni l'altérité individuelle, reconnaissant que toute collectivité et tout individu sont différence ${ }^{2}$. (Godayol 2002: 80)

Une dernière caractéristique qui a été souvent citée comme l'une des prérogatives de la traduction féminine, et qui représente l'un des traits distinctifs des bonnes traductions de textes pour la jeunesse, est le travail sur la musicalité, sur la ponctuation, dans la tentative de créer un rythme-corps qui représente en effet, dans les écritures destinées 
aux jeunes lecteurs, l'une des caractéristiques les plus significatives. Or « le rythme comment le corps-femme se déplace dans le texte à traduire - est en général un critère de beauté des traductions au féminin » (Lotbinière 1991 : 42).

6 S'il est vrai qu'une évolution historique analogue entre la traduction féminine et la littérature de jeunesse a eu lieu - si bien qu'au fur et à mesure que cette dernière s'affranchissait de sa vocation exclusivement pédagogique, la traduction féminine se libérait du rapport ancillaire qu'elle entretenait avec la voix auctoriale et avec les différentes instances éditoriales -, cette évolution a été longue, parfois délicate. C'est ce que prouve l'analyse de certaines traductions féminines de Poil de Carotte, publiées à différents moments du $\mathrm{XX}^{\mathrm{e}}$ siècle et au débutdu $\mathrm{XXI}^{\mathrm{e}}$ siècle, et qui ont trouvé une collocation éditoriale hétérogène.

\section{Poil de Carotte, un roman de rupture, un classique}

7 La publication par Jules Renard de Poil de Carotte en 1894 (Renard 1894) suit la parution de plusieurs recueils de textes courts et du premier roman de l'auteur, L'écornifleur (Renard 1892), qui avait obtenu en 1892 les faveurs de la critique. Poil de Carotte est composé de quarante-neuf brèves séquences, toutes inspirées par la chronique familiale du protagoniste, un jeune bourgeois de province dont l'âge n'est pas précisé. Le lien thématique qui unit les différents épisodes, tous indépendants du point de vue narratif, est constitué par le malaise psychologique de l'enfant dû à l'atmosphère familiale névrosée et notamment au manque d'amour maternel. Le roman, considéré comme le chef-d'œuvre de Renard, fut adapté en 1900 par l'auteur lui-même (Renard 1900), qui en tira une comédie en un acte, et ensuite par Julien Duvivier, qui en fit un film, en version muette en 1925, et en version parlante en 1932. Vingt ans plus tard, en 1952, Paul Mesnier réalisa une nouvelle adaptation cinématographique, moins théâtrale que celles de Duvivier, et en 1973 Henri Graziani eut recours, pour son Poil de Carotte à Néstor Almendros en tant que directeur de la photographie.

Le premier aspect qu'il est essentiel d'observer est la double appartenance de ce roman à la littérature censée s'adresser à un public de lecteurs adultes et à celle pensée pour de jeunes lecteurs. La première édition est publiée chez Flammarion sans illustrations, et elle fut conçue pour un lecteur adulte. L'édition suivante, qui ne paraît que huit ans plus tard (Renard 1902), c'est-à-dire après le succès de la pièce de théâtre, est en revanche enrichie de cinq épisodes et de cinquante dessins de Félix Vallotton. Initialement destiné aux adultes, ce n'est donc que plus tard que le roman fut répertorié parmi les textes s'adressant également à de jeunes lecteurs; au début du $\mathrm{XX}^{\mathrm{e}}$ siècle, il était d'ailleurs assez fréquent que des textes présentant des personnages enfants ou adolescents, deviennent tôt ou tard des classiques de jeunesse. Cette assimilation fut toutefois plus difficile pour Poil de Carotte que pour d'autres romans, et cela pour plusieurs raisons, liées essentiellement au pouvoir de rupture du roman, tant sur le plan thématique que sur le plan formel.

9 Le sujet de l'enfant malheureux n'était certes pas inédit à l'époque de Renard; les romans du XIX siècle pullulaient au contraire de petits protagonistes martyrs, pensons tout simplement au personnage de Rémy du roman Sans famille (Malot 1878) que Malot avait publié en 1878. Mais la misère de ces enfants était toujours motivée par des événements extérieurs, et les jeunes victimes de la malveillance des adultes récupéraient en général le bonheur perdu au moment où ils retrouvaient leur place au 
sein de la famille. Avant Renard, aucun écrivain n'avait eu le courage d'affirmer que la famille elle-même pouvait devenir une importante source de malheur (cf. Pitzorno 1984 : 16), que celui-ci pouvait être entièrement indépendant d'événements extérieurs, et être lié plutôt au malaise intérieur, psychologique du jeune personnage :

Precorrendo alcune delle più recenti teorie psicanalitiche e sociologiche, Renard rivela che la famiglia in quanto tale può essere un inferno, il principale focolaio di nevrosi e di infelicità senza rimedio ${ }^{3}$. (Pitzorno $1984: 16$ )

Une œuvre si féroce contre la famille, au point d'avoir été définie comme « une sorte de ferment de dissolution du fonctionnement familial ou au moins un révélateur " (Laroche 2011: 199), une histoire dénonçant si explicitement la figure maternelle, jusqu'alors point de repère obligé de toute la littérature traditionnelle, ne pouvait que susciter de fortes perplexités chez les pédagogues.

Un deuxième élément thématique "subversif » par rapport aux conventions narratives de l'époque concerne la vision de l'enfant: les personnages de Renard, et notamment Poil de Carotte, sont des enfants vrais, authentiques, sur le plan physique et psychologique. Le roman dédaigne la rhétorique de l'enfance et Renard

ne considère pas l'enfant, selon la conception la plus généralement répandue aux âges classiques, comme un petit être plein de manques et de perversité que seule la société peut amener à sa vraie maturité d'homme. Il n'en fait pas davantage le parangon de toutes les vertus naturelles brimées ou perverties par l'éducation et la vie familiale. (Autrand $1978: 194$ )

12 Passant au niveau plutôt formel de l'œuvre, nous pouvons constater son aspect novateur, d'une part en raison du manque d'évolution des événements narrés, et d'autre part grâce au mélange et à la coprésence dans le texte de passages relevant de plusieurs genres littéraires.

13 Au cours du roman, Renard se débarrasse de certaines formes narratives romantiques et réalistes qui privilégiaient la présence d'un narrateur omniscient, et il dispose le réel sous la loupe contradictoire et impitoyable d'un enfant devenant adolescent, qui commence à avoir son opinion sur les choses. L'auteur n'exprime pas de jugements, il fait en sorteque les sentiments, les névroses et les problèmes de ses jeunes personnages soient au cœur de la narration, et que le lecteur prenne conscience de l'étrangeté de certains gestes et de certains comportements : « il non detto finisce per avere più peso del raccontato " [le non-dit est en définitive plus influent que ce qui est raconté] (Pianigiani 2008: 115). Le récit, qui contribue à tourner la page du naturalisme, progresse par juxtaposition de morceaux choisis apparemment décousus, fortement fragmentés et la plupart du temps autonomes, qui forment

un livre où il y a du jeu, en équilibre instable, à la limite un livre à faire [...]. Le récit est ouvert et appelle la collaboration du lecteur : c'est un récit en partie libre. Le double sentiment est donc un sentiment de liberté : liberté de l'auteur à l'égard du lecteur, liberté du lecteur à l'égard du texte. (Autrand 1978 : $180 ; 183$ )

14 À cette hétérogénéité des épisodes racontés s'accompagne le mélange formel des genres : on passe assez brutalement, parfois à l'intérieur même d'une section, du roman à la scène de théâtre, du récit au dialogue théâtral, du roman épistolaire à l'album où les fragments narratifs pourraient se lire comme les légendes des photos d'un album. La section finale, intitulée justement "L'Album de Poil de Carotte", représente l'anticonclusion par excellence: en revenant, à travers un album photographique imaginaire, à certains clichés de l'enfance de Poil de Carotte, loin de mettre fin au récit, Renard le laisse ouvert, en en faisant « uno degli indiscussi capolavori che onorano e 
scandiscono la storia della letteratura per l'infanzia. [...] [Renard] non esita a burlarsi di tutto, a scardinare convenzioni, a deridere stereotipi. » (Faeti 2006: 239) [1'un des chefsd'œuvre incontestables qui jalonnent et ennoblissent l'histoire de la littérature de jeunesse. [...] Renard n'hésite pas à se moquer de tout, à désamorcer toute convention, à se jouer des stéréotypes].

15 C'est d'ailleurs grâce à son caractère ouvert que l'œuvre de Renard continue encore aujourd'hui à jouir, tant en France qu'en Italie, d'une collocation éditoriale différenciée, orientée vers le public adulte ainsi que vers les jeunes lecteurs, et qu'elle a eu accès à l'univers des classiques :

children's classics are often particularly refined and complex literary texts, for which it can be difficult to identify potential addressees. In some cases the author specifically wrote for an audience of children. Whereas in other cases children claimed certain texts as their own, regardless of the author's intentions. Therefore, classics often lend themselves to multiple reading levels and can be enjoyed by both adults and children ${ }^{4}$. (Pederzoli $2011: 149$ )

\section{Voyage à travers quelques traductions au féminin de Poil de Carotte : d'un roman pédagogiquement convenable à l'histoire d'une douloureuse émancipation}

16 La première traduction italienne de Poil de Carotte, significativement intitulée Il martirio di un fanciullo: Pel di Carota (Renard 1915), date de 1915. Elle est due à Édouard et Achille Macchia, et s'adresse à un public adulte. Cette édition est suivie par d'autres traductions, toutes réalisées par des traducteurs, et il faudra attendre 1945 pour voir paraître la première traduction de la main d'une femme, Maria Letizia Ascarelli ${ }^{5}$. Cette édition (Renard 1945) est, elle aussi, conçue pour un public adulte, et, du point de vue éditorial, rien n'indique qu'elle puisse être destinée à un double destinataire. Suivent d'autres traductions réalisées par des hommes, et ce n'est qu'en 1955 que Fabbri Editori (Renard 1955) décide de publier, dans la collection «I capolavori » s'adressant aux jeunes lecteurs d'une classe d'âge pourtant non mentionnée, le roman de Renard dans la traduction de Frida Ballini ${ }^{6}$, agrémenté par les illustrations en couleurs de Bartoli. En 1967, une troisième traduction au féminin (Renard 1967) est réalisée par Gina Marzetti Noventa $^{7}$, accompagnée par les illustrations en noir et blanc de Rialdo Guizzardi. Rien qu'à lire les titres des différentes sections de l'œuvre, nous percevons une différence considérable entre la traduction d'Ascarelli et les deux autres traductions illustrées. La traduction de 1945 est la seule qui, s'adressant à un destinataire adulte, traduit intégralement l'œuvre, sans aucune coupure, alors que dans la traduction de Frida Ballini seules 39 des 49 sections originales sont traduites, et dans celle de Gina Marzetti il manque 2 sections.

D'un point de vue éditorial, la traduction par Frida Ballini, très réduite par rapport à l'original, indique sur le frontispice qu'il s'agit d'une «Traduzione », alors que celle par Gina Marzetti Noventa détaille en ajoutant «Traduzione e riduzione» [Traduction et adaptation]. La première traduction raccourcit donc une partie considérable de l'œuvre : le jeune lecteur italien n'est pas confronté aux sections du roman de Renard (telles que «La taupe »), où le jeune Poil de Carotte manifeste toute son agressivité, aux sections (telles que "Honorine») où le protagoniste fait preuve d'un manque de 
sincérité et pour susciter la sympathie de sa mère induit en erreur la vieille servante Honorine, qui sera par la suite mise à la porte. Sont également éliminées des parties (telles que "Sauf votre respect » ou «Le pot »), où la mère, pour punir son fils, oblige Poil de Carotte à manger, bien incorporée à sa soupe, une partie de ses excréments ou elle oublie de lui laisser le pot en l'obligeant à faire pipi au lit. Si ces coupures n'ont pas d'effets majeurs sur l'économie générale du roman par rapport au malaise du jeune Poil de Carotte, dans la mesure où sont maintenues la plupart des autres sections qui tendent à dénoncer la pesanteur de la situation, elles n'en privent pas moins le roman d'une dimension importante, celle de l'ironie et d'un certain humour (cf. Autrand 1978). Il en résulte une œuvre narrative qui, d'une part se veut édulcorée, apprivoisée, vraisemblablement en raison d'interventions éditoriales; d'autre part, elle est mutilée d'un trait moqueur qui, dans le texte original, jouait un rôle fondamental, à savoir, précisément, celui de rendre le roman accessible à un double destinataire.

La coupure qui, du point de vue de la censure, produit un effet beaucoup plus important, est celle menant à la suppression, tant dans la traduction de Ballini que dans celle de Marzetti Noventa, de la section «Les joues rouges ». Ici, en décrivant la vie de Poil de Carotte dans une institution scolaire, Renard s'attarde sur le récit de ce qui se déroule le soir dans le dortoir, avant que les enfants ne s'endorment, et ose suggérer au lecteur un intérêt de l'instituteur Violone pour son élève Marseau. Poil de Carotte, un enfant sagace et particulièrement perméable aux émotions, après avoir vexé Marseau par l'appellatif de "pistolet » (Renard 1965 : 92), dénonce l'instituteur, qui est éloigné du collège le lendemain. Or, dans la littérature de jeunesse italienne des années cinquante et soixantes, la sexualité, et notamment l'intérêt d'un adulte pour un adolescent, était considérée comme un sujet tabou, scabreux. D'où la décision, sans doute éditoriale, de censurer ce passage. D'ailleurs, l'édition traduite par Frida Ballini supprime également la section intitulée "Mathilde », où Poil de Carotte manifeste une attirance physique pour une jeune fille de son âge.

Un dernier cas de censure qu'il importe de citer ici concerne l'avant-dernière section, "Les mots de la fin », où Poil de Carotte avoue à son père ne plus aimer sa mère et lui fait part de son désir de quitter à jamais la maison familiale. Pour que son discours soit persuasif, Poil de Carotte défie son père en lui laissant entrevoir, au cas où il essaie de l'en empêcher, la possibilité, extrêmement agressive, de son suicide. S'agissant d'un passage fondamental dans l'économie du roman, aucune édition n'a pu censurer cette séquence, mais dans l'édition Fabbri de 1955 l'allusion à la modalité du suicide, c'est-àdire la pendaison, est quand même supprimée.

La traduction-adaptation qui date de 1967, réalisée par Gina Marzetti Noventa, apparaît donc, au premier abord, moins épurée et purgée que la traduction de Frida Ballini, mais elle est caractérisée en retour par la voix de la traductrice qui oriente résolument la réception du roman à travers, en particulier, l'insertion de nombreuses notes en bas de page. Ces prises de parole, loin de souligner la complexité des rapports au sein de l'institution familiale entre les parents et les enfants, et entre les frères et les sœurs, canalisent la responsabilité de l'atmosphère invivable vers la figure maternelle, et aplatissent du coup la personnalité de Poil de Carotte, emblème d'une nouvelle vision de l'enfant que l'auteur avait voulue non plus idéalisée, mais complexe et contradictoire :

Rouge, les larmes aux yeux, [Poil de Carotte] crache sur la taupe et la jette de toutes ses forces, à bout portant, contre la pierre. (Renard $1965: 46$ ) 
Rosso in volto, le lagrime agli occhi, sputa sulla talpa e la scaglia con gran violenza sulla pietra9. (Renard 1967: 20)

Marzetti Noventa banalise également la section «La révolte ", où Poil de Carotte, après avoir vécu tant de moments de dureté et de mortification, parvient enfin, à travers un simple adverbe négatif qu'il adresse à sa mère, à se libérer du joug d'un amour filial qu'il avait perçu, jusqu'ici, comme instinctif et nécessaire, de l'autorité parentale et, pour finir, du lien de subordination maladif vis-à-vis de sa sœur et de son frère. La note de la traductrice, en cherchant des raisons extérieures au protagoniste, affaiblit la force subversive et primordiale du refus du jeune personnage d'obéir à sa mère :

\begin{tabular}{|l|l|}
\hline MADAME LEPIC & La signora Lepic: \\
Mon petit Poil de Carotte chéri, je t'en prie, tu & - Mio piccolo, caro Pel di Carota, ti prego, sii \\
serais bien mignon d'aller me chercher une livre & tanto gentile d'andarmi a cercare una libbra di \\
de beurre au moulin. Cours vite. On t'attendra & burro al mulino. Ti aspetteremo, prima di \\
pour se mettre à table. & metterci a tavola. \\
POIL DE CAROTTE & Pel di Carota: \\
Non, maman. (Renard $1965: 166)$ & - No, mamma ${ }^{10} .($ Renard $1967: 140)$ \\
\hline
\end{tabular}

En raison de ces interventions de la voix de la traductrice, cette édition italienne du roman méconnaît et néglige l'une des caractéristiques principales qui contribue au contraire à son intérêt, à savoir ses liens avec le roman de formation, lequel avait déjà commencé, dans la seconde moitié du XIX ${ }^{e}$ siècle, à se détourner des canons de la littérature destinée au public adulte, pour accéder au monde de la littérature de jeunesse, et qui témoigne, à la croisée du XIX ${ }^{e}$ et du XX $X^{e}$ siècle, d'un éloignement entre les générations :

Al bivio del Novecento la centralità dell'io nei romanzi di formazione che prendono forma sul crinale dell'ultima metamorfosi del genere, si coglie, soprattutto, nell'inconfondibile evidenza di giovinezze [...] distinte dalle precedenti per la loro vocazione alla lontananza e alla separatezza dal mondo adulto nei cui confronti vanno crescendo la diffidenza e l'incomunicabilità. [...] In quel clima che fluttua tra fine Ottocento e primi del Novecento, tra le generazioni si produce, gradualmente, una franosa perdita di contatto promossa dalla rigidità delle istituzioni e dalle tragedie annunciate e inscenate dalla società degli adulti ${ }^{11}$. (Bernardi $2011: 63-64$ ).

Cette même dimension est par contre reconnue, ou plutôt mise en valeur, par l'une des plus récentes traductions au féminin du roman de Renard, à savoir celle réalisée par Rossana Campo ${ }^{12}$, publiée en 2007 par Feltrinelli (Renard 2007) dans la collection «Universale economica I classici » et qui n'est pas spécialement orientée vers un destinataire précis ${ }^{13}$. L'analyse des stratégies de traduction adoptées par Campo révèle non seulement le respect de l'intégralité de l'œuvre, assuré par sa collocation éditoriale, mais aussi la fidélité au sens profond du texte, en particulier à la force novatrice des derniers chapitres qui

indicano con chiarezza l'inizio dell'emancipazione e la costruzione di una personalità autonoma. È ancora un ribellismo in fase predolescenziale, ma determinato a segnare un punto decisivo di non ritorno: l'acquisizione di una capacità di riconoscere le emozioni, di leggere gli eventi affettivi nella loro valenza profonda e irriducibile ${ }^{14}$. (Pianigiani $2008: 116$ )

En ce sens Rossana Campo, une écrivaine qui essaie toujours, dans son écriture à elle, de faire passer la force de la langue parlée et de faire abstraction de tout ce qui pourrait 
sembler inauthentique, arrive en traduction à trouver un espace de médiation entre les différents destinataires auxquels Renard s'adressait, ainsi qu'on peut le constater dans le passage suivant tiré de la section «Les idées personnelles » :

Pour moi, dit-il, les titres de famille ne signifient rien. [...] Le hasard vous a faits mon frère et ma sœur. Pourquoi vous en serais-je reconnaissant? À qui la faute, si nous sommes tous trois des Lepic? Vous ne pouviez l'empêcher. Inutile que je vous sache gré d'une parenté involontaire. (Renard $1965:$ 160)
Per me, i legami famigliari non significano niente. [...] È il caso che vi ha fatto nascere come miei fratelli. Perché dovrei esservene riconoscente? Di chi è la colpa se siamo tutti e tre dei Lepic? Nessuno di noi poteva farci niente. È inutile che io vi sia riconoscente di una parentela involontaria. (Renard 2007 : 133)

La collocation éditoriale de la traduction de Campo, au sein d'une collection qui publie les classiques, des textes que l'on peut donc lire à différentes étapes de la vie, lui facilite sûrement la tâche, mais le trait distinctif de son interprétation du texte de Renard est sans aucun doute l'affinité entre le refus de la part de l'auteur français de toute convention, de toute rhétorique liée à la famille, et sa résistance personnelle à elle contre les lieux communs, contre ce qui est donné pour acquis, contre toute idée reçue. De ce point de vue, la traduction publiée par Feltrinelli semble confirmer notre hypothèse initiale, c'est-à-dire le fait que les éditeurs contemporains misent de plus en plus sur la traduction féminine, espérant qu'une heureuse empathie puisse se créer entre la voix de la traductrice et la voix du texte à traduire, entre la voix de la traductrice et l'écoute, parfois différenciée en termes de classe d'âge, que l'on peut prêter au texte de départ. De ce pari dépend d'ailleurs la présence, encore insuffisante, mais grandissante, dans le marché éditorial contemporain, d'un paratexte écrit par la traductrice; dans l'édition Feltrinelli, Rossana Campo insère en postface une lecture qu'elle intitule emblématiquement, en citant une phrase de Poil de Carotte, « Non tutti hanno la fortuna di essere orfani ! » [Tout le monde n'a pas la chance d'être orphelin !] (Renard 2007 : 157-162).

L'analyse de ces traductions au féminin du roman de Jules Renard, publiées à différents moments, et au sein de collections profondément inégales, nous permet de tirer brièvement quelques conclusions. Au-delà des orientations morales et pédagogiques auxquelles Frida Ballini et Gina Marzetti Noventa se sont pliées, il reste un aspect essentiel à explorer, à savoir l'espace de médiation, que nous pourrions définir au second degré, dans lequel se sont trouvées, dans le passé, les traductrices de littérature de jeunesse par rapport aux éditeurs, aux rédacteurs. Dans ce domaine particulier, plus que dans d'autres, la ligne éditoriale a sans aucun doute orienté les stratégies de traduction, notamment en ce qui concerne les omissions, les censures, et la présence éventuelle d'une voix normalisante. L'analyse de la traduction contemporaine de Rossana Campo, qui se singularise fortement grâce aussi à sa collocation éditoriale différente, nous permet en tout cas de nous réjouir de l'imbrication étroite entre l'affranchissement de certains classiques de leur vocation exclusivement édifiante, et l'émancipation de la traduction féminine de certains stéréotypes dont les femmes ont dû, pendant très longtemps, se faire les gardiennes tutélaires. 


\section{BIBLIOGRAPHIE}

AUTRAND, Michel (1978). L'humour de Jules Renard. Paris : Klincksieck.

BERNARDI, Milena (2011). Il cassetto segreto : letteratura per l'infanzia e romanzo di formazione. Milan : Unicopli.

BRUNO, Pierre (2010). La littérature pour la jeunesse. Médiologie des pratiques et des classements. Dijon : Éditions Universitaires de Dijon.

DELISLE, Jean éd. (2002). Portraits de traductrices. Artois : Les Presses de l'Université d'Ottawa.

FAETI, Antonio (2006). « Postfazione », in Jules Renard, Pel di Carota. Milan : Fabbri, 238-240.

GODAYOL, Pilar (2002). Spazi di frontiera. Genere e traduzione. Bari : Palomar edizioni.

LAROCHE, Hugues (2011). « Poil de Carotte: initiation à la littérature rousse », Les Bulletins de l'Association « Les Amis de Jules Renard »: « Jules Renard, Un œil clair pour notre temps, Actes du colloque (26 et 27 mars 2010)», 195-205.

LOTBINIÈRE, Susanne (de) (1991). Re-belle et Infidèle/The Body Bilingual. Toronto-Montréal : Women's Press/Les éditions du remue-ménage.

MALOT, Hector (1878). Sans famille. Paris : E. Dentu.

MALOT, Hector (2001 [1878]). Senza famiglia, trad. de Rossana Guarnieri. Milan : Fabbri.

MARZETTI NOVENTA, Gina (2008). Favole di La Fontaine raccontate al femminile. Bergame : Larus.

NIÈRES-CHEVREL, Isabelle (2009). Introduction à la littérature de jeunesse. Paris : Didier.

PEDERZOLI, Roberta (2011). The «'Paratext Effect' in the Translation of Children's Classics : The Case of La guerre des boutons ", in Elena Paruolo éd., Brave New Worlds: Old and New Classics of Children's Literature. Bruxelles : Peter Lang, 147-168.

PERGAUD, Louis (2003 [1912]). La guerra dei bottoni, trad. de Angela Nanetti. Turin : Einaudi.

PIANIGIANI, Guglielmo (2008). «Ravel e Renard : Le cygne», Per leggere, 14, 113-136.

PITZORNO, Bianca (1984). «Introduzione », in Jules Renard, Pel di Carota e Storie naturali. Novara : Istituto Geografico De Agostini, 9-18.

RENARD, Jules (1892). L'écornifleur. Paris : P. Ollendorff.

RENARD, Jules (1894). Poil de Carotte. Paris : Flammarion

RENARD, Jules (1900). Poil de Carotte : comédie en 1 acte. Paris : Société d'éditions littéraires et artistiques.

RENARD, Jules (1902). Poil de Carotte; avec 50 dessins de F. Vallotton. Paris : Flammarion.

RENARD, Jules (1915). Il martirio di un fanciullo: Pel di Carota, trad. di Édouard et Achille Macchia. Naples : F. Bideri.

RENARD, Jules (1945). Pel di Carota, trad. de Maria Letizia Ascarelli. Rome : De Carlo.

RENARD, Jules (1955). Pel di Carota, trad. de Frida Ballini, illustrazioni di Bartoli. Milan : Fabbri.

RENARD, Jules (1965). Poil de Carotte. Paris : Garnier-Flammarion. 
RENARD, Jules (1967). Pel di Carota, trad. et adaptation de Gina Marzetti Noventa, illustrations de Rialdo Guizzardi. Bergame : Janus.

RENARD, Jules (1987). Pel di Carota, trad. di Anna Maria Fizzotti. Milan : Lito.

RENARD, Jules (2007). Pel di Carota, trad. di Rossana Campo. Milan : Feltrinelli.

\section{NOTES}

1. Le critique Pierre Bruno témoigne très bien de la difficulté, encore actuelle, de définir précisément la littérature de jeunesse : si d'un côté il est assez aisé de classifier les publications destinées à un public de jeunes en partant de leur collocation éditoriale, d'autre part une définition ne se basant que sur le destinataire apparaît lacunaire, d'autant plus que le lexème 'de jeunesse' ne semble pas trouver un accord inconditionné : "Pour une large part impossible à définir, la "littérature pour la jeunesse" reste, pis encore, difficile à nommer. La divergence des termes (littérature enfantine, littérature pour la jeunesse, littérature d'enfance et de jeunesse, littérature jeunesse, littérature de jeunesse...) témoigne tout d'abord de l'évolution de son public ou, pour être plus précis, des classifications dominantes des populations juvéniles selon les époques. La tendance est tout à la fois à une subdivision croissante des cibles commerciales, donc des tranches d'âge, donc des termes et, dans le langage courant, à une substitution du terme de jeunesse à celui d'enfance, dominant naguère » (Bruno 2010 : 20). Devant forcément choisir, nous utiliserons dans la présente étude l'expression « littérature de jeunesse ».

2. C'est nous qui traduisons en français à partir de l'édition italienne du texte de Godayol.

3. «Renard, devançant les théories psychanalytiques et sociologiques les plus récentes, nous révèle que la famille, en soi, peut s'avérer invivable, et qu'elle peut devenir un foyer de névrose et de malheur inéluctables » (notre traduction).

4. «Les classiques pour la jeunesse sont souvent des textes extrêmement recherchés et complexes du point de vue littéraire, qui peuvent, dès lors, poser des problèmes d'identification des destinataires possibles. C'est parfois l'auteur qui a choisi expressément d'écrire pour un public de jeunes, alors que, dans d'autres cas, ce sont les jeunes lecteurs qui revendiquent certains textes comme faisant partie de la littérature qui leur est destinée, indépendamment des intentions de l'auteur. Les classiques se prêtent souvent à plusieurs niveaux de lecture, et ils peuvent donc être appréciés aussi bien par les adultes que par les jeunes lecteurs » (notre traduction).

5. La traductrice réalisa, toujours en 1945, une deuxième traduction publiée par De Carlo éditeur, la même maison d'édition qui publia Poil de Carotte: elle traduisit Reigen de Schnitzler, avant de disparaître définitivement du monde éditorial.

6. L'activité de traduction de Frida Ballini a été importante et constante, des années cinquante jusqu'à aujourd'hui (sa dernière traduction publiée date de 2010) ; Poil de carotte est cependant le seul ouvrage qu'elle ait traduit du français, toutes ses autres traductions ayant été réalisées à partir de l'anglais. Parmi ses auteurs d'élection Charles Dickens, Rudyard Kipling, Oscar Wilde, Eleanor Hodgman Porter, Louisa May Alcott.

7. Gina Marzetti Noventa aborde le monde de l'écriture à la fin des années quarante en écrivant des textes didactiques pour les dernières classes de l'école primaire ; elle passe ensuite à l'écriture des textes de fiction pour un public de jeunes lecteurs en suivant un double parcours, celui de la création personnelle et celui de la réécriture de classiques déjà existants, comme, par exemple Le avventure di Pinocchio ou Mille e una notte. Quant à son activité en tant que traductrice, elle traduit également de l'anglais, mais elle se consacre surtout aux classiques de la littérature française, dont Sans famille. Une particularité qui mérite d'être mentionnée est la 
publication en 2008, par Larus, des Fables de La Fontaine «racontées au féminin par Gina Marzetti Noventa » (Marzetti Noventa : 2008).

8. La section est encore supprimée dans la traduction d'Anna Maria Fizzotti, publiée en 1987 par Lito, avec des illustrations de Cecile Anguera (Renard 1987).

9. Note en bas de page: «Pel di Carota piange. Quasi sempre la sua sensibilità si manifesta proprio nel momento in cui compie un'azione crudele. Sono impulsi d'un fanciullo infelice, combattuto tra tendenze buone e cattive. Ed è questo il risultato di un'educazione che ha fatto di tutto per sopprimere in lui ogni personalità ». [Poil de Carotte pleure. Sa sensibilité se manifeste presque toujours au moment où il accomplit un geste agressif. Ses instincts sont ceux d'un enfant malheureux, tiraillé entre des pulsions de douceur et d'agressivité. Et cela est dû à une éducation qui a tout fait pour briser chez lui toute forme de personnalité]. (notre traduction)

10. Note en bas de page: « Forse sono le eccessive e forzate espressioni di tenerezza della madre che irritano Pel di Carota e lo spingono alla ribellione » [Ce sont les manifestations de tendresse de la mère, excessives et affectées, qui agacent Poil de Carotte, en l'amenant à la révolte]. (notre traduction)

11. «Au carrefour du vingtième siècle, la prépondérance du je dans les romans de formation qui s'ébauchent sur le créneau de la dernière métamorphose de ce genre littéraire, est reconnaissable notamment dans les figures des jeunes personnages [...] ; ceux-ci diffèrent de leurs prédécesseurs car ils sont destinés à s'éloigner et à se séparer du monde des adultes, à l'égard duquel ils éprouvent une méfiance et une incommunicabilité grandissantes. [...] Dans ce climat fluctuant entre la fin du XIXe et le début du XXe siècle, le contact entre les générations tend à diminuer, voire à disparaître, sous l'effet de la dureté des institutions et des tragédies annoncées et mises en scène par la société des adultes ». (notre traduction)

12. Rossana Campo (1963-) est une romancière italienne vivant entre la France et l'Italie, souvent associée aux écrivains définis vers la moitié des années 1990 comme "cannibales " à cause du réalisme souvent très cru de leurs romans. Le noyau narratif des histoires romanesques de Campo est souvent représenté par un univers féminin complexe et contradictoire, qui recherche, à partir des conquêtes passées du féminisme, de nouvelles formes d'existence individuelle. La tentative de l'auteure de recréer, dans l'espace de la page écrite, la force et l'intensité de la langue parlée, se traduit stylistiquement par un important travail sur le rythme de l'écriture, souvent dépourvue de signes de ponctuation.

13. Les classiques de jeunesse publiés au sein de cette collection, tels que, par exemple, Alice nel paese delle meraviglie, Cuore, Robinson Crusoe, I viaggi di Gulliver, ne sont pas, en général, agrémentés par des illustrations, mais évoquent, grâce à d'autres choix éditoriaux, tels que, par exemple, le choix de l'image pour la couverture, la possibilité d'un double destinataire.

14. « indiquent clairement l'exorde d'une émancipation et la construction d'une personnalité indépendante. Il s'agit d'une insubordination encore préadolescentielle, mais décidée à marquer un point de non-retour : la conquête de la capacité à reconnaître ses propres émotions, à lire les événements émotifs en raison de leur portée profonde et irrécusable » (note traduction).

\section{RÉSUMÉS}

Dans cette étude, après une présentation des questions traductologiques concernant, dans le monde éditorial contemporain, la traduction au féminin de la littérature de jeunesse, nous menons une analyse d'un corpus de trois traductions italiennes «féminines » du roman Poil de 
Carotte (1894) de Jules Renard, datant respectivement de 1955, 1967 et 2007. À travers une analyse comparative de quelques aspects de la traduction, tels que les omissions et les censures, la présence de la voix des traductrices, nous suivons le parcours de la réception italienne du roman, d'abord interprété comme une œuvre qu'il fallait censurer pour qu'elle puisse s'adresser à un double destinataire, jeune et adulte, ensuite réévalué comme un roman de formation et de douloureuse émancipation du jeune protagoniste.

After a presentation of translation aspects that concern, in the contemporary publishing world, the translation of children's literature by women, this study analyzes a corpus of Italian "female" translations of the French novel Poil de Carotte (1894) by Jules Renard, published in 1955, 1967 and 2007. Through a comparative analysis of some aspects of the examined translations such as omission and censorship, the presence of the female translator's voice, the study investigates the reception of the novel in Italy, where it was initially interpreted as a novel to be censored because of its double reader - youth and adults - and subsequently reassessed as a Buildungsroman with the painful emancipation of the protagonist.

\section{INDEX}

Mots-clés : Littérature de jeunesse, Monde de l'édition, Traduction au féminin, Double destinataire, Roman de formation

Keywords : Children's and young adults' literature, Editorial target, Gender translation, Double reader, Buildungsroman

\section{AUTEUR}

\section{CHIARA ELEFANTE}

Université de Bologne-Forlì 